\title{
Domain formation on the nonpolar lithium niobate surfaces under electron-beam irradiation: A review*
}

\author{
T. R. Volk ${ }^{\dagger}$, , L. S. Kokhanchik ${ }^{\star}$, R. V. Gainutdinov ${ }^{\dagger}$, Y. V. Bodnarchuk ${ }^{\dagger}$ and S. D. Lavrov ${ }^{\S}$ \\ ${ }^{\dagger}$ Shubnikov Institute of Crystallography of Federal Scientific Research Centre \\ "Crystallography and Photonics" of Russian Academy of Sciences \\ Moscow 119333, Russia \\ Institute of Microelectronics Technology and High Purity Materials \\ Russian Academy of Science, Moscow 142432, Russia \\ $\S_{\text {Moscow Technological University (MIREA), Moscow 119454, Russia }}$ \\ qvolk-1234@yandex.ru
}

Received 12 January 2018; Revised 13 February 2018; Accepted 26 February 2018; Published 2 April 2018

\begin{abstract}
In this review, our recent results on the electron-beam domain writing (EBDW) on the nonpolar surfaces of $\mathrm{LiNbO}_{3}$ crystals of different compositions are presented. Under EB irradiation of the nonpolar surfaces, domains nucleated in irradiation points grow frontally along the polar $Z$-direction in a thin (of microns in thickness) surface layer; the driving force is the tangential component of space-charge fields induced by EB in irradiation points. This geometry of the experiment provides a possibility of three-dimensional (3D) characterization of domain patterns using the combination of atomic force microscopy (AFM) and second harmonic generation (SHG) confocal microscopy methods. The obtained results permitted us to relate the main characteristics of domain formation (the domain sizes and velocity $V_{f}$ of the frontal motion) to the irradiation conditions (the accelerating voltage $U$ of scanning electron microscopy (SEM), EB current $I$, the inserted charge $Q$ ). The domain depth $T_{d}$ is controlled by $U$ via the electron penetration depth; the domain length $L_{d}$ increases linearly with $Q$ owing to the domain frontal growth by the viscous friction law. The electron emission coefficient $\sigma$ affects the domain formation due to the fundamental dependence of $\sigma$ on $U$. In the framework of current approach to EB charging of insulators, the effect of an enhanced conductance on EBDW characteristics is analyzed. The difference between EBDW characteristics observed in varied $\mathrm{LiNbO}_{3}$ compositions is discussed in the framework of the intrinsic defect structure of $\mathrm{LiNbO}_{3}$. The obtained results extend the possibility of EBDW application to a wider range of crystals.
\end{abstract}

Keywords: Domains; electron beam; lithium niobate.

\section{Introduction}

As known, regular domain patterns (periodically poled structures) can serve for optical-frequency conversion on the basis of quasi-phase matching (QPM) principle, following from the fundamental relation between the nonlinear quadratic susceptibility and spontaneous polarization. The most attractive material for nonlinear-optical convertors of this type is $\mathrm{LiNbO}_{3}$ because of a unique combination of the proper optical parameters. The relevant bibliography can be found in some recent monographs. ${ }^{1,2}$ Potentials of regular domain patterns for various applications have triggered a wide search for methods of domain patterning. The standard method is the application of an external field to an electrode pattern deposited onto the polar crystal surface. This method meets some limitations ${ }^{1}$ related to the backswitching effect and domain merging at the electrode edges.

An alternative approach is provided by the domain writing by an electron beam (EB) of scanning electron microscopy (SEM), which offers certain advantages over the field method due to the fact that, first, this noncontact method imposes no restrictions on the domain sizes and periods, which in the case of external-field technique are confined by the domain merging, and, second, there is no evidences (at least in $\mathrm{LiNbO}_{3}$ ) of the domain backswitching on EB turning-off. Additionally, what is directly related to the subject of the given paper, the EB domain writing (EBDW) provides a possibility to fabricate domains on the nonpolar surfaces of ferroelectric crystals, which is of interest for some integrated schemes on $\mathrm{LiNbO}_{3}$ nonpolar surfaces (e.g., Ref. 3).

There is a large amount of works on EBDW on the polar crystal surfaces; in this case, a domain nucleated in the irradiation point grows axially along the polar axis inside the crystal bulk. Some characteristics of EBDW on the polar $\mathrm{LiNbO}_{3}$ surfaces are summarized in our recent publication. ${ }^{4}$ The generated domains are needle-shaped and shoot through the sample thicknesses up to $0.5 \mathrm{~mm}$. The mechanism of this axial growth is still under debate, partly due to missing characteristics (such as velocities of the frontal and sideways

\footnotetext{
*Selected paper from The International Workshop on Advanced Dielectric Materials and Their Applications, Xi'an, China, Oct. 8-11, 2017.
}

This is an Open Access article published by World Scientific Publishing Company. It is distributed under the terms of the Creative Commons Attribution 4.0 (CC-BY) License. Further distribution of this work is permitted, provided the original work is properly cited. 
domain growth) of this process. The authors of Ref. 5 relate the EB-induced frontal domain growth to the mechanism of ferroelectric breakdown. ${ }^{6}$ An uncertainty in the interpretation of the domain axial growth is due partly to difficulties in the three-dimensional (3D) characterization of occurring domains, specifically of their depth inside the crystal bulk.

In the given paper, we summarize our recent results ${ }^{4,7-13}$ on EBDW on the nonpolar $\mathrm{LiNbO}_{3}$ surfaces. In addition to some above-mentioned practical aspects, the convenience of this EBDW geometry for experimental studies is due to the fact that the written domains are accessible for 3D characterization, ${ }^{8}$ which permits us to discuss the mechanism of domain growth. Further, we describe the dependence of domain formation on irradiation conditions and some material characteristics. The consideration is based on the current concept of EB charging of insulators, formulated, e.g., Refs. 14 and 15.

The scheme of domain formation under EB incident onto a nonpolar surface of a ferroelectric crystal is illustrated in Fig. 1. Domains nucleate in irradiation points and grow frontally along the polar axis in a thin (within few microns) surface layer. These planar domains are needle-shaped similarly to those appeared under EB irradiation of the polar surfaces. A scenario similar to that shown in Fig. 1 was observed in the pioneering experiments on domain fabrication on the nonpolar surfaces by applying high dc-voltages to a needle-like electrode contacting the $Y$-surface of $\mathrm{LiNbO}_{3}$ crystal (Ref. 16 and references therein) and when domain writing by the biased conductive tip of scanning force microscope on the nonpolar $\mathrm{LiNbO}_{3}$ surface (Ref. 17 and references therein). EBDW on the nonpolar surface was realized for the first time by the author of the given paper (L. K.) in $\mathrm{LiTaO}_{3}$ crystals. ${ }^{18}$ Provided that the distance between irradiation points along the polar axis is within certain length specified by the irradiation conditions, the domains arising in the successive irradiation points are merging

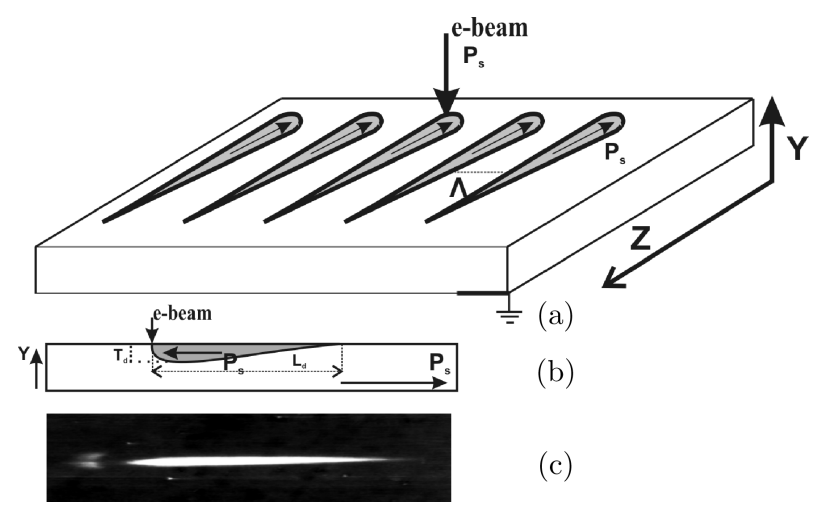

Fig. 1. The scheme of EBDW on the nonpolar surface of a ferroelectric crystal: (a) writing of a domain "comb" by point-to-point displacement of $\mathrm{EB}$ ( $\Lambda$ is the distance between irradiation points); (b) the $X$-cross-section of a written domain, illustrating a decrease of the domain depth $T_{d}$ along the polar axis and (c) an example of PFM image of EB-written domains.

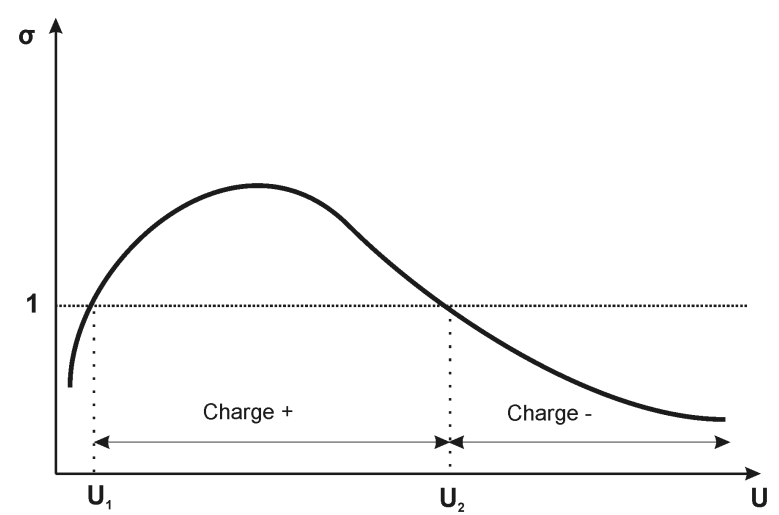

Fig. 2. The general presentation of the total electron emission as a function of the EB energy $U$ in insulators; $\sigma$ is the total electron emission coefficient composed of the electron backscattering and secondary-electron emission. The regions Charge + and Chargecorrespond to positive and negative surface charging, respectively, and beam energies $U_{1}$ and $U_{2}$ corresponding to $\sigma=1$ characterize the charge equilibrium state.

to form an elongated quasi-continuous domain. Based on this important feature, the two-dimensional (2D) EBDW technique was elaborated ${ }^{18}$ which permitted to fabricate the domain gratings as large as $600 \times 600 \mu \mathrm{m}^{2}$.

Both under external fields and EB irradiation, the driving force of domain growth is the tangential component $E_{r}(z)$ (where $r$ is the cylindrical coordinate in the $X$-surface) of a "point" field, which in our case is a space-charge field pumped by EB in irradiation point.

We now shortly describe the phenomena occurring under EB irradiation of an insulator, ${ }^{14,15}$ illustrated by the scheme presented in Fig. 2.

EB irradiation induces a space-charge field in the irradiation point, whose formation is determined by the irradiation characteristics. These are the accelerating voltage $U$ of SEM and EB current $I$, the inserted charge $Q=I t_{\text {irr }}$ and irradiation dose $D=Q / S$ (where $t_{\text {irr }}$ and $S$ are the exposure time and irradiation area, respectively). The material property contributing to the space charge is the total electron emission coefficient $\sigma$. Figure 2 illustrates schematically the EBinduced electron emission and its dependence on the EB energy.

The space-charge kinetics involves trapping, detrapping and diffusion of electrons, and for given exposure conditions can be presented by the following generalized expression:

$$
Q_{\mathrm{sc}}(t)=Q_{\mathrm{sat}}\left[1-\exp \left(-t / \tau_{\mathrm{eff}}\right)\right]
$$

where $Q_{\text {sat }}$ is the equilibrium negative space- charge value. The effective time constant $\tau_{\text {eff }}$ describes the time required for the trapping and detrapping process to reach equilibrium. This empirical parameter depends both on the irradiation conditions and material properties. All other conditions being equal, $\tau_{\text {eff }} \approx 1 / U$. An equilibrium charge state in highresistant dielectrics such as $\mathrm{LiNbO}_{3}$ is attained in tens or 
hundreds of ms. The kinetics of $Q_{\mathrm{sc}}$ decay on EB turning-off is given by the following expression:

$$
Q_{\mathrm{sc}}(t)=Q_{\mathrm{sat}}\left[\exp \left(-t / \tau_{M}\right)\right]
$$

where $\tau_{M}=\varepsilon \varepsilon_{0} / G$ is the dielectric relaxation time, $\varepsilon \varepsilon_{0}$ and $G$ are the dielectric constant and dark conductivity, respectively.

The value of $Q_{\text {sat }}$ is governed by the total balance of currents

$$
I=I_{Q}+I_{L}+I_{\sigma}
$$

where $I$ is the incident EB current, $I_{Q}=d Q_{\mathrm{sc}} / d t$ is the displacement current related to electron trapping/detrapping, $I_{L}$ is the leakage current consisting of a bulk $I_{L}^{\text {bulk }}$ and surface $I_{L}^{\text {surf }}$ components, and $I_{\sigma}$ is the total emission current. The displacement current $I_{Q}$ is determined by the scheme of involved electron traps. The bulk leakage current $I_{L}^{\text {bulk }}$ depends on the radiation-induced conductivity $G_{i}$, proportional to the dark conductivity $\mathrm{G}$. The surface component $I_{L}^{\text {surf }}$ in $\mathrm{LiNbO}_{3}$ was shown to be neglected. ${ }^{4}$ The emission current $I_{\sigma}$ is summed up of the emission of secondary and backscattered electrons. For the equilibrium charge state, the contribution from electron emission can be presented approximately as

$$
Q_{\text {sat }} \sim I t_{\text {irr }}(1-\sigma) .
$$

Emphasizing, for any comparative estimates, this expression can be applied exclusively in allied materials having an identical $\sigma(U)$ curve (Fig. 2).

Equations (1)-(4) underlie the dependence of the domain formation on the scheme of electron traps and related parameters. At the same time, the accelerating voltage $U$ controlling $Q_{\mathrm{sc}}(t)$ via $\tau_{\text {eff }}(\mathrm{Eq}$. (1)) contributes to the equilibrium $Q_{\text {sat }}$ solely through the emission coefficient $\sigma$ (Fig. 2).

Further, in the framework of this qualitative consideration, we will analyze the relation of EB-induced domain evolution to the factors controlling the space-charge field. The domain motion under EBDW is characterized by the domain length $L_{d}$ along the polar axis, the domain width $W_{d}$ and the domain depth $T_{d}$ (or, which is the same, the domain thickness) in the irradiation point (Fig. 1). An important characteristic is the average velocity of domain frontal growth, which can be presented as $V_{f}=d L_{d} / d t_{\text {irr }}$ assuming the charge equilibrium to be achieved.

\section{The Samples and Experimental Techniques}

The samples under study were optically polished $Y$-cut $\mathrm{LiNbO}_{3}$ plates of different compositions. The following compositions were investigated and compared: a nominally pure congruent $\mathrm{LiNbO}_{3}(\mathrm{CLN}) ; \mathrm{LiNbO}_{3}$ doped with 4 at.\% and 7.5 at.\% $\mathrm{Zn}$ (designated below as LN-4Zn and LN-7.5Zn, respectively); a chemically reduced $\mathrm{LiNbO}_{3}$ denoted as RLN. The selection of these compositions was motivated by the following reasons. $\mathrm{LiNbO}_{3}: \mathrm{Zn}$ was chosen due to the wellknown effects of $\mathrm{Zn}$ impurity on the $\mathrm{LiNbO}_{3}$ intrinsic defect structure. ${ }^{1}$ The specified $\mathrm{Zn}$ concentrations will be explained as follows.

EBDW in RLN answers the question of how the domain formation is affected by the conductivity increase, since the chemical reduction enhances the $\mathrm{LiNbO}_{3}$ conductivity. ${ }^{1}$ In particular, RLN samples under study were obtained by annealing a CLN plate in hydrogen at $550^{\circ} \mathrm{C}$, which according to Ref. 19 raises $G$ up to $10^{-11}$ to $10^{-13} \mathrm{Ohm}^{-1} \mathrm{~cm}^{-1}$ compared to $G=\left(10^{-16}-10^{-18}\right) \mathrm{Ohm}^{-1} \mathrm{~cm}^{-1}$ in undoped CLN. ${ }^{1}$

Domain writing was performed in a JSM-840A SEM equipped with the NanoMaker program which allowed us to control EB scanning over the surface and the irradiation dose. Experiments were carried out at SEM acceleration voltages $U$ from $5 \mathrm{kV}$ to $25 \mathrm{kV}$, EB currents in the range from $0.1 \mathrm{nA}$ to $3 \mathrm{nA}$ and exposure times $t_{\text {irr }}$ in the range from $10 \mathrm{~ms}$ to $1-2 \mathrm{~s}$; the local irradiation area was $S=0.5 \mu \mathrm{m}^{2}$. The current $I$ was controlled with a Faraday cup located adjacent to the sample. Using the scheme described in Ref. 11, the total electron emission coefficient $\sigma$ was evaluated. Other experimental details can be found in Refs. $7-10$. On $+Y$ and $-Y$ surfaces, we wrote isolated (single) domains and regular domain arrays denoted below as "combs" (Fig. 1), fabricated by means of point-to-point discrete displacement of EB along the $X$-direction with specified distances $\Lambda$ between the irradiation points, the exposure time $t_{\text {irr }}$ being constant for a given comb. The values of $L_{d}$ and $W_{d}$ for given exposure conditions ( $U, I$ and $t_{\text {irr }}$ ) were averaged over the comb.

The domain sizes $L_{d}, W_{d}$ and $T_{d}$ were measured using the combination of piezo-response force microscopy (PFM) in the in-plane mode of operation, second harmonic generation confocal microscopy (SHGCM) on reflection and selective chemical etching.

PFM images were obtained by means of measuring the electromechanical response signal:

$$
H_{\omega}=\left[\frac{1}{k} \frac{d C}{d z}\left(\frac{V^{\uparrow}+V^{\downarrow}}{2}\right) \pm d_{15}\right] U_{\mathrm{ac}},
$$

where $k$ is the force constant of the tip, $C$ is the tip-sample capacity, $\frac{V \uparrow+V \downarrow}{2}$ is the average contact potential difference between the tip and the crystal surface, and $U_{\text {ac }}$ is the ac voltage between the tip and the electroded counter surface of the crystal. Si probes with Pt/Ti conducting coating (CSC21, MikroMasch, Estonia) were used; tip radius $R \leq 40 \mathrm{~nm}$, cantilever stiffness $k \sim 0.12 \mathrm{~N} / \mathrm{m}$ (A lever) and $k \sim 2 \mathrm{~N} / \mathrm{m}$ (B lever) and resonance frequencies $f \sim 12 \mathrm{kHz}$ (A lever) and $f \sim 105 \mathrm{kHz}$ (B lever). All atomic force microscopy (AFM) experiments were carried out with an NTEGRA PRIMA AFM (NT-MDT, Russia).

The fundamentals of domain observations by the SHGCM are presented in Refs. 20-22. The approach of Refs. 20-22 based on the Kleynman-Boyd theory ${ }^{23}$ was elaborated for the transmission geometry. We extended this approach to the reflection geometry of $\mathrm{SHGCM}^{8}$ taking into account the small domain thickness of about microns, which would not 


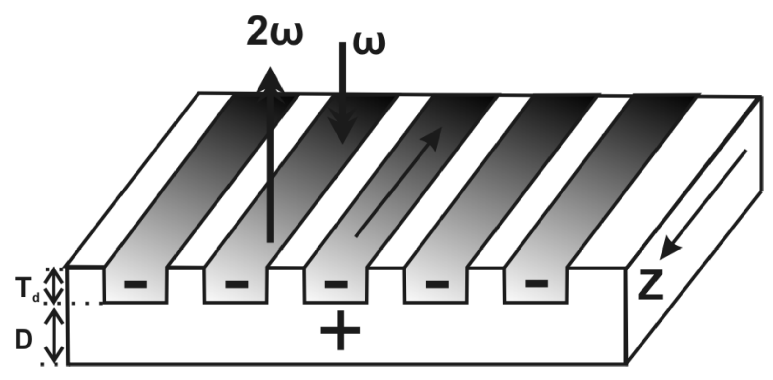

Fig. 3. The scheme of SHG conversion on domains in the reflection geometry. $T_{d}$ and $D$ are the thicknesses of domains and crystal plate, respectively, $D \gg T_{d}$.

permit to detect the contrast against the background of the crystal thickness of $0.5 \mathrm{~mm}$.

The scheme of SHGCM is presented in Fig. 3. The pump was generated by a Ti-sapphire laser (TiF-100, Avesta project, Russia) with the wavelength $800 \mathrm{~nm}$ at the repetition rate of $100 \mathrm{MHz}$; the pulse average power and duration were $70 \mathrm{~mW}$ (on the sample) and $100 \mathrm{fs}$, respectively. Focusing of the pumping beam and detection of the reflected SH beam were performed with a confocal microscope (alpha300s+, WITec, Germany). Other details can be found in Ref. 8.

Selective chemical etching was performed in a hot acid solution $\mathrm{HF}+2 \mathrm{HNO}_{3}$. As the $-Y$ surface in $\mathrm{LiNbO}_{3}$ etches much faster than the $+Y$ one,${ }^{24}$ the domains after etching appear as long triangular hillocks and grooves on the $-Y$ and $+Y$ surfaces, respectively. The height of hillocks was estimated using an Axioplan 2 optical interference microscope. The depth of grooves was measured by the contact AFM operation mode.

\section{Experimental Results and Discussion}

To start with, emphasize that all EB-written domain patterns discussed below are completely stable and reveal no degradation in real time up to months and more.

As shown below, the domain formation under EB irradiation is notably affected by the $\mathrm{LiNbO}_{3}$ composition because of a variety of reasons. In particular, the equilibrium charge $Q_{\text {sat }}$ which determines the driving field of the domain motion depends on the electron emission coefficient $\sigma$ (Eq. (4)), which in its turn is governed by the accelerating voltage $U$. Figure 4 presents the experimental data on $\sigma$ versus $U$ in the samples under study. ${ }^{11}$ In the first approximation, these $\sigma$ values do not depend on $\mathrm{LiNbO}_{3}$ composition.

\subsection{The "starting" irradiation conditions of domain appearance}

Domains appear at certain "starting" conditions $\left(U_{\mathrm{st}}, I_{\mathrm{st}}, Q_{\mathrm{st}}\right.$ and $D_{\text {st }}$ ). Note that these conditions for the nonpolar $\mathrm{LiNbO}_{3}$ surfaces discussed in this section notably differ from those on the polar surfaces. The available data for EBDW on the polar surface in $\mathrm{LiNbO}_{3}$ were summarized in our recent publication. $^{4}$

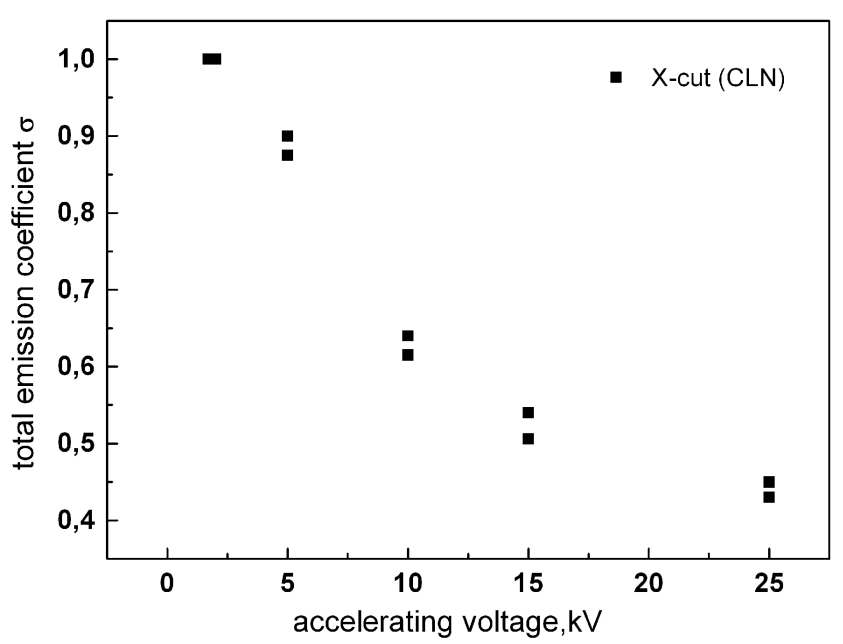

Fig. 4. The secondary electron emission coefficient $\sigma$ versus accelerating voltage obtained in CLN crystals under study.

According to the results presented in Refs. 7-11, on the nonpolar CLN surfaces, domains appear after irradiation by EB with $U \geq 5 \mathrm{kV}, I \geq 0.1 \mathrm{nA}$, the current densities being $\geq 2 * 10^{10} \mathrm{pA} / \mathrm{cm}^{2}$. The starting inserted charge and dose are, respectively, $Q_{\mathrm{st}} \approx 5 \mathrm{pC}$ and $D_{\mathrm{st}} \approx 1000 \mu \mathrm{C} / \mathrm{cm}^{2}$. Close characteristics are observed in LN-4.5Zn. In RLN, domains are formed by $U \geq 10 \mathrm{kV}, I \geq 1 \mathrm{nA}$ with the current density of $2 * 10^{11} \mathrm{pA} / \mathrm{cm}^{2}$; the starting characteristics are $Q_{\mathrm{st}} \approx 25 \mathrm{pC}$ and $D_{\mathrm{st}} \approx 5000 \mu \mathrm{C} / \mathrm{cm}^{2}$, i.e., by a factor of 5 higher than in CLN. ${ }^{4}$ In highly doped LN-7.5Zn, domains appear at $Q_{\mathrm{st}}$ of about $300 \mathrm{pC}$.

In terms of a simplified approach presented by Eqs. (1)-(3), an increase of $Q_{\mathrm{st}}$ and $D_{\mathrm{st}}$ in RLN and LN-7.5Zn with respect to CLN is owing to a modified balance equation (3). As the emission coefficients $\sigma$ in all compositions are very close, so must be the equilibrium emission current $I_{\sigma}$. An increase of $Q_{\mathrm{st}}$ in RLN by a factor of 5 suggests that $Q$ values in these compositions are lower at least by a factor of 5 compared to CLN, all things being equal, which in the framework of Eq. (3) is qualitatively accounted for by an enhanced leakage current $I_{L}^{\text {bulk }}$ related to an increased $G$. Further, we will speculate about the microscopic origin of increasing $G$.

So far, EBDW was used almost solely in the very highresistant $\mathrm{LiNbO}_{3}$ and $\mathrm{LiTaO}_{3}$. The main conclusion drawn from studies of EBDW in RLN is that the primary parameter determining the domain emergence is the EB current density, that is applying EBDW to crystals with varied conductance requires matching of this parameter. This conclusion paves the way to extend the number of ferroelectric crystals in which this method can be realized.

\subsection{The domain depth $T_{d}$}

To estimate the domain depth $T_{d}$, the samples were chemically etched after EBDW, whereupon the surface was scanned 


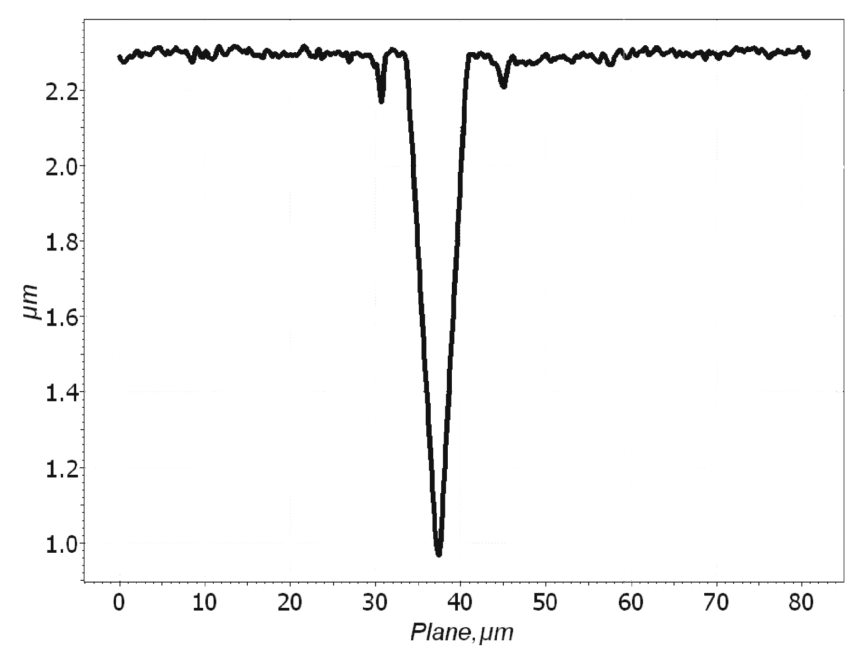

Fig. 5. Profile of an etch groove along the $X$-axis after EBDW on the $Y$-surface $(U=15 \mathrm{kV})$.

using the contact AFM operation mode. Figure 5 gives an example of the profile of an etch groove along the $X$-axis.

Our consideration presented in detail in Refs. $8-11$ permitted us to conclude that the domain depth in the irradiation point is determined by the penetration depth $R_{e}$ of the primary electrons. The equilibrium $R_{e}$ in solids is presented by a generalized power function $R_{e}=A U_{0}^{k} / \rho$, where $U_{0}$ is the initial electron energy, and $\rho$ is the crystal density. From a comparison of $T_{d}$ values obtained for different $U$ by the chemical etching, to the calculated $R_{e}(U)$ for $\mathrm{LiNbO}_{3}$ taken from the literature, we inferred the following dependence of the domain depth on the accelerating voltage for CLN crystals under study:

$$
T_{d} \cong 79 U^{1.7} / \rho,
$$

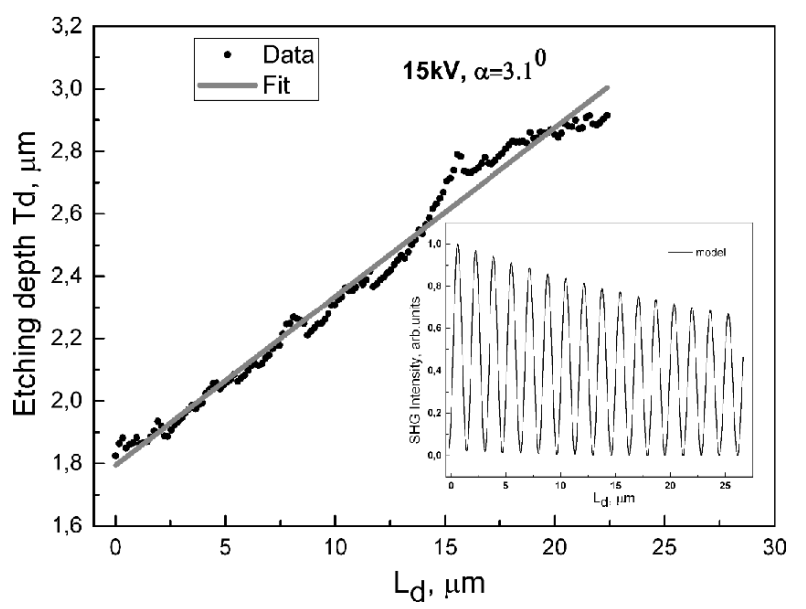

(a) where $\rho=4.65 \mathrm{~g} / \mathrm{cm}^{3}$ for CLN. These results provide a calibration curve, which permits one to specify $T_{d}$ by matching the accelerating voltage $U$. The validity of this approach was confirmed experimentally both in bulk crystals ${ }^{8-11}$ and He-implanted optical waveguides on the nonpolar $\mathrm{LiNbO}_{3}$ surfaces. $^{12}$

By analogy with the case of in-plane domain motion under AFM-tip voltages applied to the nonpolar crystal surfaces, ${ }^{25}$ the EB-induced field decreases with the distance from the irradiation point, so the domain thickness $T_{d}$ is expected to lowered along the polar axis. To verify this assumption, isolated domains were written by varied accelerating voltages, other irradiation conditions being equal. The samples were chemically etched, whereupon the depth of the etched grooves along the domain axis was measured by the contact AFM.

Figures 6(a) and 6(b) exemplify the etching profiles obtained by this technique. These plots are fitted by linear functions (solid lines in Fig. 6) except for the areas around the irradiation points (the upper parts of the graphs), where the domain shape is smeared because of EB scattering. These results show that a written domain can be represented as a wedge with an angle $\alpha$ specified by the ratio $T_{d} / L_{d}$. In the case shown in Figs. 6(a) and 6(b), the maximum depths of domains written by $U=15 \mathrm{kV}$ and $10 \mathrm{kV}\left(t_{p}=150 \mathrm{~ms}, I=100 \mathrm{pA}\right)$ are $T_{d}=1.1 \mu \mathrm{m}$ and $0.65 \mu \mathrm{m}$, respectively; the calculated angles of etching profiles are $\alpha=3.1^{\circ}$ and $2.2^{\circ}$. Since $T_{d}$ and $L_{d}$ are controlled by $U$ and $t_{\text {irr }}$, respectively, the wedge-angle can be specified by the irradiation conditions.

The wedge-like shape of domains accounts for the discontinuity of SH intensity $I_{2 \omega}(z)$ along the domain axis observed when examination of EB-written domains by SHGCM technique (Fig. 7(a)). ${ }^{8}$ Unlike SHGCM images, the PFM images of domains are unbroken (Fig. 7(c)), which evidence that domains emerge completely at the surface,

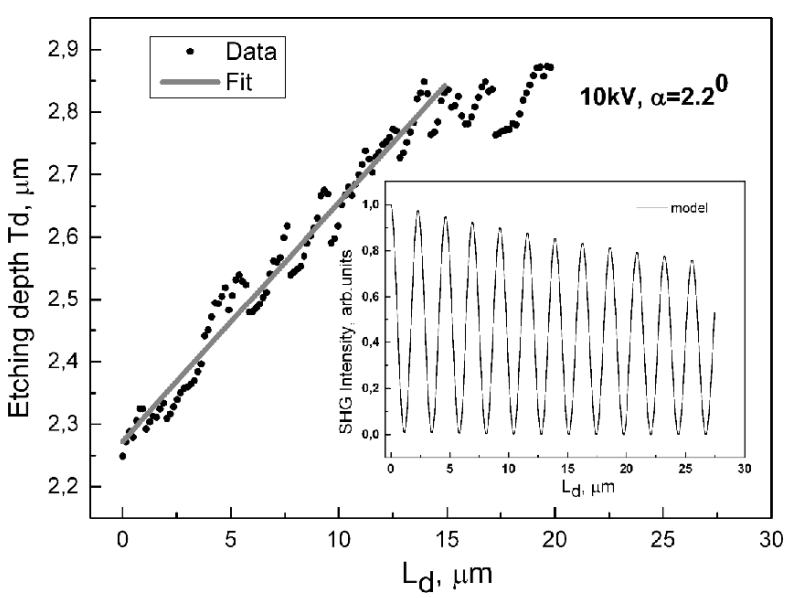

(b)

Fig. 6. (a, b). Etching profiles (dotted) of two isolated domains written by $15 \mathrm{kV}$ and $10 \mathrm{kV}$, respectively $\left(t_{p}=150 \mathrm{~ms}, I=100 \mathrm{pA}\right)$; the maximum etching depths occur in the irradiation areas (the upper parts of the graphs). The solid lines show fitting by linear functions; the evaluated wedge angles are $3.1^{\circ}$ and $2.2^{\circ}$, respectively. The insets present the distribution of SH intensity versus domain length $I_{2 \omega}(z)$ calculated by Eq. (7). 

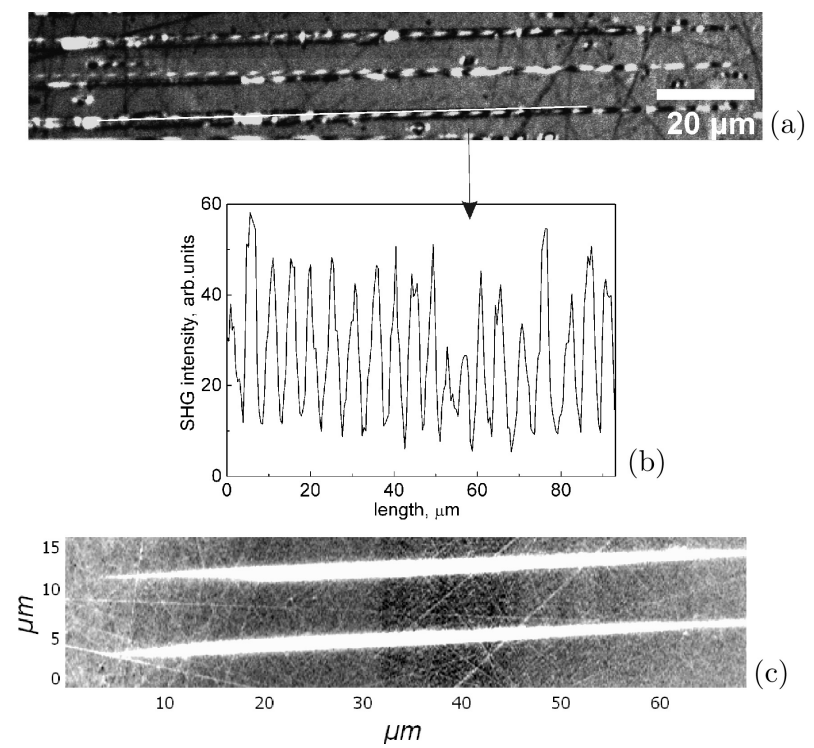

Fig. 7. (a, b) SHGCM and (c) PFM images of EB-written domain patterns.

i.e., a discontinuous $I_{2 \omega}(z)$ could not be attributed to a partial subsurface "dip" of domains.

The effect of the wedge-like domain shape on the $I_{2 \omega}(z)$ distribution in the written domain gratings was analyzed in the framework of the approach proposed by us for SHGCM on reflection. ${ }^{8}$ For this case, the total SHG field can be presented by the classical expression:

$$
\begin{aligned}
E_{2 \omega} \propto & \left(\chi_{1} \int_{-y_{f}}^{-y_{f}+T_{d}} \frac{e^{(i * \Delta k * y)}}{1+i * y / y_{r}} d y\right) \\
& +\left(\chi_{2} \int_{-y_{f}-T_{d}}^{-y_{f}+D} \frac{e^{(i * \Delta k * y)}}{1+i * \frac{y}{y_{r}}} d y\right)
\end{aligned}
$$

where $T_{d}$ and $D$ are the domain depth and crystal thickness, respectively, $E_{2 \omega}$ is the electric field of a spherical harmonics (SH) wave, $y_{f}$ is the distance of the focus position from the crystal surface, $y_{r}$ is the Rayleigh length, $\chi_{1}$ and $\chi_{2}$ are nonlinear susceptibilities of the inverted domains and initial (single-domain) crystal matrix, respectively; $\chi_{2}=$ $-\chi_{1}=\chi_{33}$ (in the given geometry). The SH intensity is $I_{2 \omega}=\left(E_{2 \omega}\right)^{2}$. Based on Eq. (7), the SH intensity versus domain length $I_{2 \omega}(z)$ was calculated for domains, whose etching profiles are shown in Fig. 6. The corresponding $I_{2 \omega}(z)$ curves are shown in Fig. 6 in the insets. One can see the oscillating $\mathrm{SH}$ intensity, the oscillation period decreasing with increasing $T_{d}$. These results could help to estimate the domain depth (thickness) from the $\mathrm{SH}$ intensity distribution.

\subsection{The domain length $L_{d}$}

The domain length was estimated from PFM images of domains, exemplified by Fig. 1(c). Figures 8(a) and 8(b) compare the plots $L_{d}$ versus exposure times for CLN, RLN and $\mathrm{LN}-4 \mathrm{Zn}$. The common property of the graphs $L_{d}\left(t_{\mathrm{irr}}\right)$ is their linearity, which is observed in the whole range of $U$ from $5 \mathrm{kV}$ to $25 \mathrm{kV}$ and $I$ from $0.1 \mathrm{nA}$ to $1 \mathrm{nA}$ and even in the range of $t_{\text {irr }}$ up to minutes. ${ }^{7}$ As well, the linear $L_{d}\left(t_{\text {irr }}\right)$ plots were observed in domain gratings and He-implanted waveguides. ${ }^{12}$ Assuming the charge equilibrium to be achieved, this linearity was interpreted ${ }^{7}$ as the result of domain frontal growth by the viscous friction law:

$$
V_{f}=\mu E,
$$

where $V_{f}=d L_{d} / d t_{\text {irr }}$ is the average velocity of domain frontal growth, $\mu$ is the domain-wall (DW) frontal mobility and $E$ is the driving field. This law is known to describe the domain frontal growth under high external fields. ${ }^{26}$ It predicts the domain length to be linear with $t$ ( $L=V_{f} t$ where $t$ is the time of field application), which actually occurs in our case.

The slopes of linear $L_{d}\left(t_{\text {irr }}\right.$ as well as $L_{d}$ values depend on the composition (all factors being the same), as seen from comparison of the data for CLN and LN-4Zn (Fig. 8(a)) and CLN and RLN (Fig. 8(b)). $L_{d}$ in RLN is drastically lesser than in CLN (Fig. 8(b)), which similarly to an increase of the starting values $Q_{\text {st }}$ and $D_{\text {st }}$ in RLN (Sec. 3.1) originates obviously from an enhanced leakage current $I_{L}^{\text {bulk }}$ caused by an increased conductivity $G$ in RLN. As a result, the driving field $E$ in Eq. (8) is reduced. As shown below in Sec. 3.4, an increased $G$ is not the only reason of very small domains in RLN.

The probable microscopic origin of different $L_{d}\left(t_{\text {irr }}\right)$ in CLN and LN-4Zn (Fig. 8(a)) will be discussed in Sec. 3.4.

We now consider the plots $L_{d}\left(t_{\text {irr }}\right)$ obtained in CLN at different accelerating voltages (Fig. 8(c)). One can see a similarity between $L_{d}\left(t_{\text {irr }}\right)$ for $U=10 \mathrm{kV}$ and $15 \mathrm{kV}$, whereas $L_{d}$ for $U=5 \mathrm{kV}$ are (2-2.5) times lesser, all factors being the same. As follows from Eq. (1), the space-charge field in saturation is not dependent on $U$, so the identity of $L_{d}\left(t_{\text {irr }}\right)$ for all $U$ is expected. The small sizes of domains written by $U=5 \mathrm{kV}$ might be attributed to an enhanced surface conductivity, since under $U=5 \mathrm{kV}$ domains are formed in a thin layer of $200-250 \mathrm{~nm}$ thick. However, the reduced domain lengths in this case originate from another reason related to the specificity of electron emission. As seen from Fig. 4, the electron emission coefficient $\sigma$ increases significantly at $U=5 \mathrm{kV}$. Substituting the experimental values of $\sigma$ (Fig. 4) to the simplified expression $Q_{\text {sat }} \sim I t_{\text {irr }}(1-\sigma)$, we evaluated the $Q_{\text {sat }}$ for 5,10 and $15 \mathrm{kV}\left(I=100 \mathrm{pA}, t_{\text {irr }}=200 \mathrm{~ms}\right)$. These calculations give $Q_{\text {sat }} \approx(1.5-2) \mathrm{pC}$ for $U=5 \mathrm{kV}$ and $\approx(4.5-5) \mathrm{pC}$ for $U=10 \mathrm{kV}$. So, $Q_{\text {sat }}$ for $U=5 \mathrm{kV}$ is by a factor (2.5-3) lower than for $10 \mathrm{kV}$, which is in a very good agreement with the above-mentioned relationship between the $L_{d}$ values for $5 \mathrm{kV}$ and $10 \mathrm{kV}$ (Fig. 8(c)). At the same time, the plots $L_{d}\left(t_{\text {irr }}\right)$ for $U=10 \mathrm{kV}$ and $15 \mathrm{kV}$ are almost identical due to an almost equal $\sigma$. Strongly decreased domain sizes in thin layers and films compared to the bulk samples are obviously a general property of EBDW. 


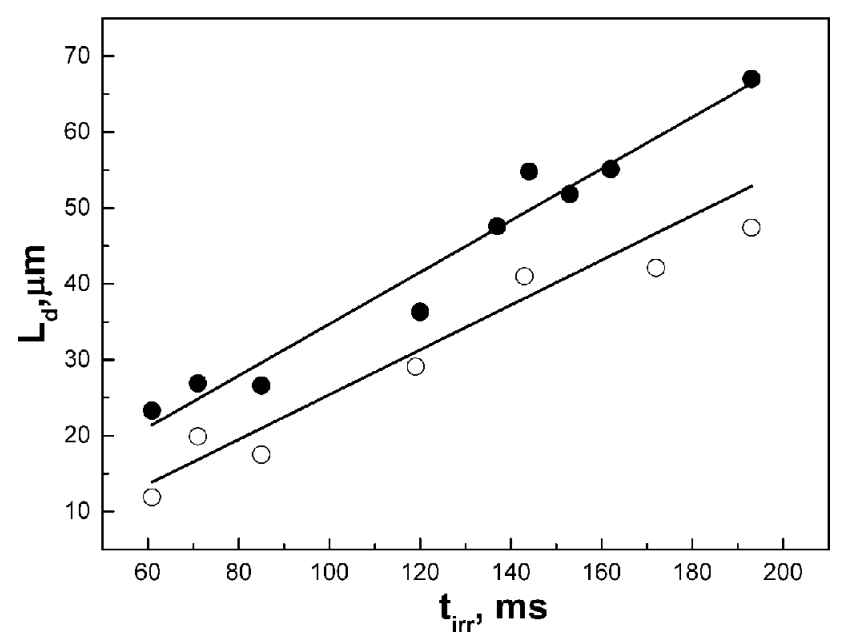

(a)

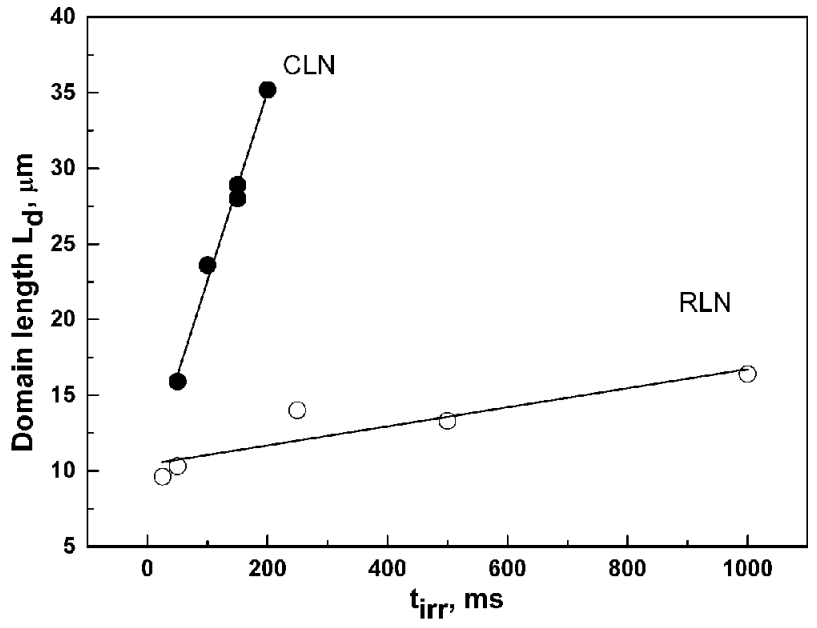

(b)

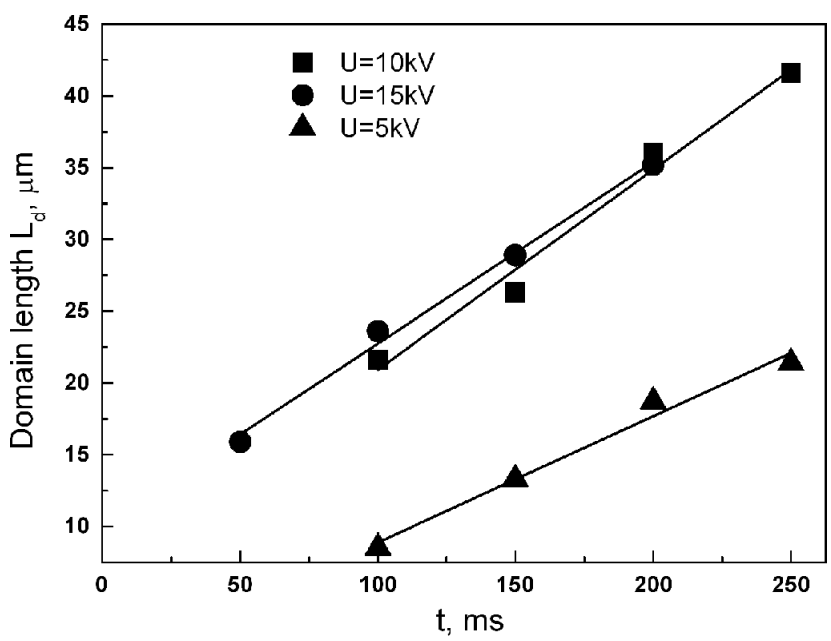

(c)

Fig. 8. Domain lengths versus exposure times in $\mathrm{LiNbO}_{3}$ crystals under study: (a) $L_{d}\left(t_{\text {irr }}\right)$ in $\mathrm{LN}-4 \mathrm{Zn}$ (upper) and CLN (lower) $(U=25 \mathrm{kV}$, $I=100 \mathrm{nA}$ ); (b) $L_{d}\left(t_{\text {irr }}\right)$ in CLN (upper) and RLN (lower); the plots were obtained at $I=100 \mathrm{pA}$ and $1 \mathrm{nA}$ for CLN and RLN, respectively $(U=15 \mathrm{kV})$ and (c) $L_{d}\left(t_{\text {irr }}\right)$ in CLN for $U=15,10$ and $5 \mathrm{kV}$ (circles, squares and triangles, respectively; $I=100 \mathrm{pA}$ ). In all cases, the solid lines show fitting by linear functions.

Note, a good fit of the data on domain sizes to the simplified evaluations of $Q_{\text {sat }}$ evidences in favor of our assumption that in all cases under study, the domain motion occurs under conditions of the charge equilibrium.

In the end of this section, we dwell very briefly on the specificity of domain length in domain gratings. ${ }^{7-10}$ Figure 9 compares $L_{d}\left(t_{\text {irr }}\right)$ for single domains and domain "combs"; the scheme of writing a comb with a period $\Lambda$ is presented in Fig. 1(a). Figures 9(a) and 9(b) present the results of writing single domains and combs with $\Lambda=10 \mu \mathrm{m}$ in CLN and RLN, respectively. In CLN (Fig. 9(a)), the average $L_{d}$ in the comb (the upper plot) exceeds notably $L_{d}$ of single domains (the lower plot), $t_{\text {irr }}$ being equal. By contrast, in RLN (Fig. 9(b)), $L_{d}$ of single domains (the squares) and $L_{d}$ in the comb (the circles) are almost identical. This specificity is explained in the following way. ${ }^{7}$

The storage time of EB-induced point space-charges $Q_{\mathrm{sc}}$ in irradiation points is determined by the dielectric relaxation time $\tau_{M}=\varepsilon \varepsilon_{0} / G$. In CLN $G=\left(10^{-16}-10^{-18}\right) \mathrm{Ohm}^{-1} \mathrm{~cm}^{-1}$, i.e., $\tau_{M} \geq 10^{4}-10^{6} \mathrm{~s}$; in the RLN samples under study, $G=\left(10^{-11}-10^{-13}\right) \mathrm{Ohm}^{-1} \mathrm{~cm}^{-1}$ (see above), which gives $\tau_{M} \leq 100 \mathrm{~s}$. The total recording time $t_{\text {rec }}$ of a comb is within hundreds of seconds. As in CLN $\tau_{M} \gg t_{\text {rec }}$, so in the course of writing of a comb, the charges $Q_{\mathrm{sc}}$ persist without decay in all irradiation points. As a result, the grating is produced by the total field of point charges $\mathbf{E}=\sum_{i=1}^{n} \mathbf{E}_{i}$ (where $E_{i}$ is a point space-charge field), which according to the viscous friction law (8) results in a larger $L_{d}$ in a grating (Fig. 9(a)). 


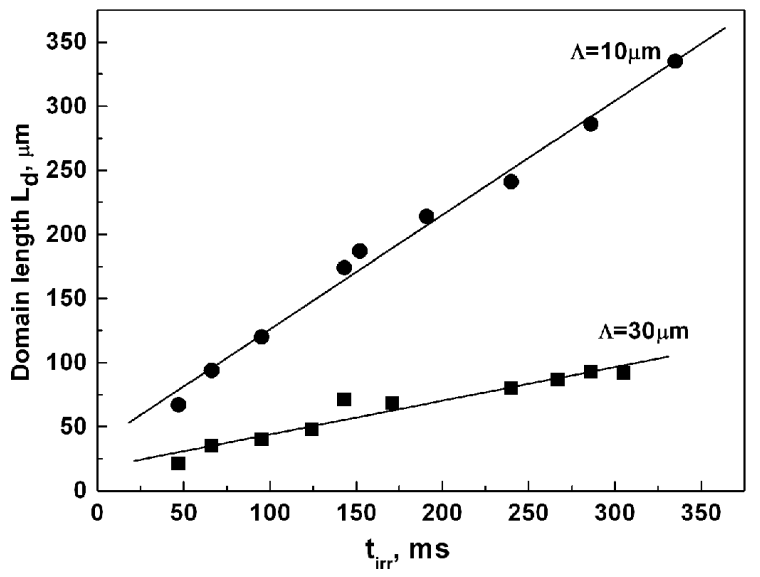

(a)

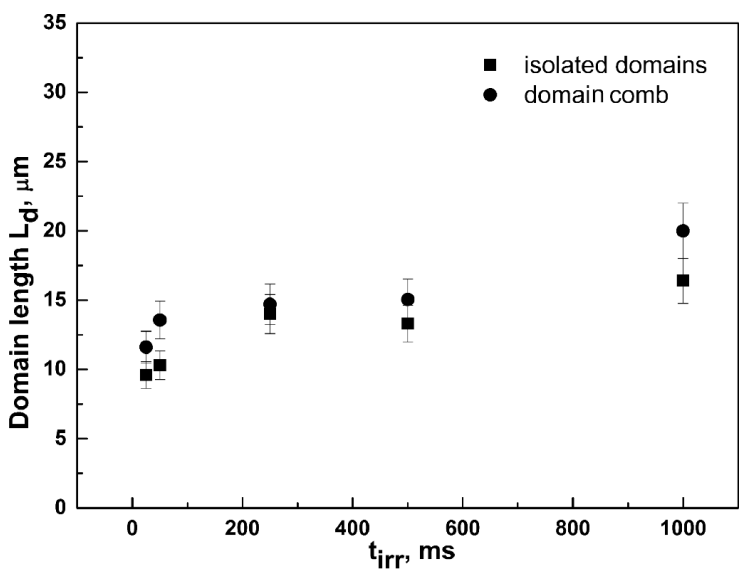

(b)

Fig. 9. A comparison of domain lengths $L_{d}\left(t_{\text {irr }}\right)$ of single domains and domain combs (written by the scheme shown in Fig. 1(a)): (a) $L_{d}\left(t_{\text {irr }}\right)$ for single domains (lower) and a comb with the period $\Lambda=10 \mu \mathrm{m}$ (upper) in CLN and (b) $L_{d}\left(t_{\text {irr }}\right)$ for single domains (squares) and a comb with $\Lambda=10 \mu \mathrm{m}$ (circles) in RLN.

By contrast, in RLN, $\tau_{M}$ is comparable to $t_{\mathrm{rec}}$, so, a relatively fast decay of point $Q_{\text {sc }}$ prevents from summing $E_{i}$ over irradiation points and the length of single domains coincide with that in the comb (Fig. 9(b)). The assumption that in CLN, the length of domain gratings is determined by the total field of point charges was supported experimentally. ${ }^{7}$ In a simplified presentation, the strength of the field $E_{z}$ at a distance $L_{d}$ from the row of $n$ point charges $Q_{\text {sc }}$, aligned parallel to $X$-axis, looks as

$$
\begin{aligned}
E & =\sum E_{i}=\sum \frac{2 Q L_{d}}{4 \pi \varepsilon \varepsilon_{0}\left[L_{d}^{2}+\left(n_{i} \Lambda\right)^{2}\right]^{3 / 2}} \\
& =\frac{Q L_{d}}{2 \pi \varepsilon \varepsilon_{0}} \sum_{i=0}^{n} \frac{1}{\left[L_{d}^{2}+\left(n_{i} \Lambda\right)^{2}\right]^{3 / 2}} .
\end{aligned}
$$

According to Eq. (9), $L_{d}$ in a grating grows with decreasing grating period $\Lambda$. The gratings with $\Lambda=10 \mu \mathrm{m}$ and $30 \mu \mathrm{m}$ were written on the $Y$-surface of CLN. ${ }^{7}$ The ratio of $L_{d}$ in these gratings was in a good agreement with the calculations by Eq. (9).

\section{4. $E B D W$ in the context of intrinsic defect structure of $\mathrm{LiNbO}_{3}$}

As seen from the foregoing, the EBDW characteristics in $\mathrm{LiNbO}_{3}$ compositions under study are different. Specifically, in RLN and LN-7.5 in particular, the starting conditions of the domain appearance $Q_{\text {st }}$ and $D_{\text {st }}$ are significantly larger; the domain lengths $L_{d}\left(t_{\text {irr }}\right)$ in all samples differ widely. We discuss the microscopic origin of this difference in the framework of the intrinsic defect structure of $\mathrm{LiNbO}_{3}$; the appropriate bibliography can be found, e.g., in Ref. 1. According to the currently accepted model, ${ }^{27}$ in Li-deficient $\mathrm{LiNbO}_{3}$ (CLN among them), the charge transport is determined by the intrinsic defects Nb-on-Li-sites $\left(\mathrm{Nb}_{\mathrm{Li}}^{4}\right.$ or
Nb-antisites) serving as the electron traps; the charge neutrality is provided by the compensation of $\mathrm{Nb}_{\mathrm{Li}}$ with $4 \mathrm{Li}$ vacancies $\left(V_{\mathrm{Li}}\right)$. Additionally, the intrinsic defect clusters $\left[\mathrm{Nb}_{\mathrm{Li}}^{4}-4 V_{\mathrm{Li}}^{\prime}\right]$ are the most probable pinning centers for DWs in $\mathrm{CLN}^{28} \mathrm{So}$, in $\mathrm{LiNbO}_{3}$, the intrinsic defect structure is responsible both for the charge transport and domain motion.

In Zn-doped crystals, $\mathrm{Zn}$ ions incorporate the Li-sites, substituting for $\mathrm{Nb}_{\mathrm{Li}}$ up to certain "threshold" concentration of 5-6 at.\% Zn. At higher $\mathrm{Zn}$ concentrations, $\mathrm{Nb}$-antisites are completely removed from the lattice, so $\mathrm{Zn}$ ions incorporate both $\mathrm{Li}$ - and $\mathrm{Nb}$-cation sites. As a consequence, the freedom from the electron traps $\mathrm{Nb}$-antisites at $[\mathrm{Zn}]$ above the threshold leads to an abrupt increase of the conductivity. The samples LN-4Zn and LN-7.5Zn belong to the below- and above-threshold compositions, respectively.

As seen from Fig. 8(a), in LN-4Zn, the domain length is notably larger than in CLN. It cannot be explained in terms of the conductivity variation, since the conductivity of $\mathrm{Zn}$-doped CLN is somewhat larger than $G$ of CLN even at low Zn concentrations. ${ }^{1}$ In terms of Eq. (8), an increased $L_{d}$ in LN$4 \mathrm{Zn}$ is accounted for by an increased DW mobility $\mu$, which is due to a reduced concentration of DW pinning centers $\left[\mathrm{Nb}_{\mathrm{Li}}^{4 \cdot}-4 \mathrm{~V}_{\mathrm{Li}}^{\prime}\right]^{28}$ Interestingly, the coercive field $E_{c}$ in $\mathrm{Zn}$ doped $\mathrm{LiNbO}_{3}$ is decreased compared to CLN. ${ }^{29}$ This effect is in line with the inference about an increased DW mobility.

Drastically increased starting conditions of the domain appearance $Q_{\text {st }}$ and $D_{\text {st }}$ in the above-threshold LN-7.5 are obviously related to a drastic increase of the conductance $G$, which, in its turn is due to a change of the intrinsic defect structure, namely, to the disappearance of $\mathrm{Nb}$-antisites.

In RLN, the scheme underlying the mechanism of conductivity suggests that a moderate reduction annealing of CLN is followed by the formation of a stable pair of bonded electrons localized on the adjacent lattice sites, the so-called bipolaron $\left(\mathrm{Nb}_{\mathrm{Nb}}-\mathrm{Nb}_{\mathrm{Li}}\right)^{2-}$. Now, instead of the electron 
trapping by $\mathrm{Nb}_{\mathrm{Li}}$, the most probable is its capture by $\mathrm{Nb}_{\mathrm{Nb}}$ to form an unstable polaron of a small radius, $\mathrm{Nb}_{\mathrm{Nb}}^{4+}$. An enhanced conductivity of RLN is accounted for by this scheme.

The increased values of $Q_{\text {st }}$ and $D_{\text {st }}$ in RLN assigned to a large $G$ are again related to a reconstruction of the intrinsic defect structure. As well, in terms of Eq. (8), the main reason of a decreased $L_{d}$ in RLN is an increased $G$. However, a detailed examination of $L_{d}\left(t_{\text {irr }}\right)$ in RLN permits us to detect an additional microscopic reason of a drastic $L_{d}$ shortening. Estimates of the average velocity $V_{f}=d L_{d} / d t$ of the frontal growth from $L_{d}\left(t_{\text {irr }}\right)$ (Fig. $8(\mathrm{~b})$ ) give $V_{f} \approx 6 \mu \mathrm{m} / \mathrm{s}$ and $\approx 130 \mu \mathrm{m} / \mathrm{s}$ for RLN and CLN, respectively, i.e., $V_{f}$ in RLN is more than by a factor of 20 lesser than in CLN. At first glance, this low $V_{f}$ is caused by a reduced driving field $E$ in Eq. (8). However, since the value of starting $Q_{\text {st }}$ in RLN exceeds that in CLN by a factor of 5 (see Sec. 3.1), one could expect the space-charge fields in RLN to be lowered by a factor of 5, too, (say, by an order of magnitude) compared to CLN. So, a much larger difference in $V_{f}$ cannot be accounted for by an increased $G$ only. In the framework of the viscousfriction law (8), an enormous difference between $V_{f}$ in RLN and CLN is indicative of a significant decrease of DW mobility $\mu$ in RLN. This conclusion seems to be completely reasonable in the context of the defect structure, since the chemical reduction destroying the clusters $\left[\mathrm{Nb}_{\mathrm{Li}}^{4 \cdot}-4 \mathrm{~V}_{\mathrm{Li}}^{\prime}\right]$ modifies obviously the pinning centers of DWs. We believe that the domain "shortening" in RLN is caused both by a decrease of the driving field due to an enhanced $G$ and by a decrease of DW mobility related to modified pinning centers.

To end this section, we mention one more experimental observation linking the processes occurring under EB irradiation with the defect structure. Examination of $L_{d}\left(t_{\text {irr }}\right)$ for different $U$ in CLN (Fig. 8(c)) leads us to an unexpected conclusion. As shown above, the fields $E$ driving domains under $U=5 \mathrm{kV}$ and $10 \mathrm{kV}$ differ by a factor of 2.5-3. However, regardless of different driving fields, the values of velocity $V_{f}$ are very close for all $U$, namely, $V_{f}=(100-$ 130) $\mu \mathrm{m} / \mathrm{s}$ for $U=5 \mathrm{kV}$ and (140-150) $\mu \mathrm{m} / \mathrm{s}$ for $U=10 \mathrm{kV}$ and $15 \mathrm{kV}$. In terms of Eq. (8), the plausible explanation of this paradox is a larger DW mobility $\mu$ under $U=5 \mathrm{kV}$. In other words, the domains formed by $U=5 \mathrm{kV}$ in a thin layer of 200-250 nm thick are more "mobile" than those formed under $U=10 \mathrm{kV}$ and $15 \mathrm{kV}$ in the layers of $1-1.5 \mu \mathrm{m}$ thick. It is reasonable to suggest that in a surface layer, an increased $\mu$ is due to a decreased concentration of DW pinning centers, i.e., of $\mathrm{Nb}$-antisites. This simple reasoning agrees with indirect evidences of an increased surface conductance with respect to the bulk in $\mathrm{LiNbO}_{3}$.

\section{Conclusion}

The results on the EBDW on the nonpolar surfaces of $\mathrm{LiNbO}_{3}$ crystals of different compositions are summarized. The crystals under study were the CLN, $\mathrm{LiNbO}_{3}$ doped with $4.5 \%$ $\mathrm{Zn}$ and $7.5 \% \mathrm{Zn}$ (i.e., the concentrations below and above the optical-damage threshold) and chemically RLN. The approach to EBDW on the nonpolar surfaces was developed, which permits us to specify the characteristics of written domain patterns by matching the irradiation conditions, particularly, the accelerating voltage $U$ of SEM, exposure time $t_{\text {irr }}$, and EB current density $Q$. By varying $U$ from $5 \mathrm{kV}$ to $25 \mathrm{kV}$, the domain depth $T_{d}$ can be specified in the range from $200-250 \mathrm{~nm}$ to $4-5 \mu \mathrm{m}$. The domain length $L_{d}$ is controlled by the exposure time $t_{\text {irr }}$, the dependence $L_{d}\left(t_{\text {irr }}\right)$ being linear in the whole range of $t_{\text {irr }}$ in all crystals under study. A contribution from the electron emission coefficient $\sigma$ to the domain formation under low $U$ was found, which is due to an enhanced emission from surface layers. The results obtained in RLN have shown that to realize EBDW in crystals with an enhanced conductivity, the matching of EB current density is required.

Different EBDW characteristics in various $\mathrm{LiNbO}_{3}$ compositions were observed. This difference is due to the modification of both charge-transport scheme and DW motion, which in their turn are determined by the intrinsic defect structure, in particular, Nb-antisites and related defect clusters.

\section{Acknowledgments}

This work was supported by the Federal Agency of Scientific Organizations (Agreement No. 007-State Task/3363/26) in part of AFM experiments and by the Russian Science Foundation (Project No. 16-0200439a) in part of SEM experiments and analysis of the experimental data. The equipment of the Shared Research Center supported by the Ministry of Education and Science (Project No. RFMEFI62114X0005) was used in experiments.

\section{References}

${ }^{1}$ T. Volk and M. Woehlecke, Lithium Niobate: Defects, Photorefraction and Ferroelectric Switching (Springer-Verlag, Berlin Heidelberg, 2008).

${ }^{2}$ S. Mailis, L.-H. Peng, S. Grilli, M. Marangoni, E. Fazio et al., Ferroelectric Crystals for Photonic Applications, eds. P. Ferraro, S. Grilli and P. De Natali (Springer-Verlag, Berlin Heidelberg, 2009), pp. 1-132.

${ }^{3}$ F. Généreux, G. Baldenberger, B. Bourliaguet and R. Vallée, Deep periodic domain inversions in xx-cut $\mathrm{LiNbO}_{3}$ and its use for second harmonic generation near $1.5 \mu \mathrm{m}$, Appl. Phys. Lett. 91, 231112 (2007).

${ }^{4}$ L. S. Kokhanchik, Y. V. Bodnarchuk and T. R. Volk, Electron beam domain writing in reduced $\mathrm{LiNbO}_{3}$ crystals, J. Appl. Phys. 122, 104105 (2017).

${ }^{5}$ L. Mateos, L. E. Bausa and M. O. Ramırez, Two-dimensional ferroelectric domain patterns in $\mathrm{Yb}^{3+}$ optically active $\mathrm{LiNbO}_{3}$ fabricated by direct electron beam writing, Appl. Phys. Lett. 102, 042910 (2013).

${ }^{6}$ M. Molotskii, Y. Rosenwaks and G. Rosenman, Ferroelectric domain breakdown, Annu. Rev. Mater. Res. 37, 271 (2007). 
${ }^{7}$ L. S. Kokhanchik and T. R. Volk, Domain inversion in $\mathrm{LiNbO}_{3}$ and $\mathrm{Zn}$-doped $\mathrm{LiNbO}_{3}$ crystals by the electron irradiation of the nonpolar Y-cut, Appl. Phys. B 110, 367 (2013).

${ }^{8}$ L. S. Kokhanchik, R. V. Gainutdinov, E. D. Mishina, S. D. Lavrov and T. R. Volk, Characterization of electron-beam recorded microdomain patterns on the nonpolar surface of $\mathrm{LiNbO}_{3}$ crystal by nondestructive methods, Appl. Phys. Lett. 105, 142901 (2014).

${ }^{9}$ L. S. Kokhanchik, R. V. Gainutdinov, S. D. Lavrov, E. D. Mishina and T. R. Volk, Electron-beam recording of domain structures on the nonpolar surfaces of $\mathrm{LiNbO}_{3}$ crystals at different SEM voltages, Ferroelectrics 480, 49 (2015).

${ }^{10}$ L. S. Kokhanchik, R. V. Gainutdinov, S. D. Lavrov and T. R. Volk, Charactristics of microdomains and microdomain patterns recorded by electron-beam irradiation on Y-cut $\mathrm{LiNbO}_{3}$ crystals, J. Appl. Phys. 118, 072001 (2015).

${ }^{11}$ L. S. Kokhanchik, R. V. Gainutdinov and T. R. Volk, Electron beam recording of microdomains on the nonpolar $\mathrm{LiNbO}_{3}$ crystal surface at different SEM accelerating voltages, Phys. Solid State 57, 949 (2015).

${ }^{12}$ T. R. Volk, L. S. Kokhanchik, R. V. Gainutdinov, Y. V. Bodnarchuk, S. M. Shandarov, M. V. Borodin, S. D. Lavrov, H. L. Liu and F. Chen, Microdoamin patterns recorded by an electron beam in He-implanted optical waveguides on $\mathrm{X}$-cut $\mathrm{LiNbO}_{3}$ crystals, IEEE J. Lightw. Technol. 33, 4762 (2015).

${ }^{13}$ S. D. Lavrov, L. S. Kokhanchik, R. V. Gainutdinov, A. S. Elshin, Y. V. Bodnarchuk, E. D. Mishina and T. R. Volk, Nonlinear-optical characterization of planar domain patterns written in $\mathrm{LiNbO}_{3}$ by electron-beam irradiation, Opt. Mater. 75, 325 (2018).

${ }^{14}$ J. Cazaux, Some consideration on the electric field induced in insulators by electron bombardment, J. Appl. Phys. 59, 1418 (1986).

${ }^{15}$ E. I. Rau, E. N. Evstafeva and M. V. Andrianov, Mechanisms of charging of insulators under irradiation with medium-energy electron beams, Phys. Solid State 50, 621 (2008).

${ }^{16}$ V. G. Zalessky and S. O. Fregatov, Micrometer-scale ferroelectric domain formation and injection of space charge in Y-cut $\mathrm{LiNbO}_{3}$ crystals, Physica B 371, 158 (2006).

${ }^{17}$ A. V. Ievlev, D. O. Alikin, A. N. Morozovska, O. V. Varenyk, E. A. Eliseev, A. L. Kholkin, V. Y. Shur and S. V. Kalinin, Symmetry breaking and electrical frustration during tip-induced polarization switching in the nonpolar cut of $\mathrm{LiNbO}_{3}$ single crystals, ACS Nano 9, 769 (2015).

${ }^{18}$ L. S. Kokhanchik and D. V. Punegov, Microscale domain structure formation by e-beam point writing on the $\mathrm{Y}$ cut surfaces of $\mathrm{LiTaO}_{3}$ crystals, Ferroelectrics 373, 69 (2008).

${ }^{19}$ P. F. Bordui, D. H. Jundt, E. M. Standifer, R. G. Norwood, R. L. Sawin and J. D. Galipeau, Chemically reduced $\mathrm{LiNbO}_{3}$ single crystals: Processing, properties and improved surface acoustic wave device fabrication and performance, J. Appl. Phys. 85, 3766 (1999).

${ }^{20}$ Y. Uesu, H. Yokota, S. Kawado, J. Kaneshiro, S. Kurimura and N. Kato, Three-dimensional observations of periodically poled domains in a $\mathrm{LiTaO}_{3}$ quasiphase matching crystal by second harmonic generation tomography, Appl. Phys. Lett. 91, 182904 (2007).

${ }^{21}$ J. Kaneshiro, S. Kawado, H. Yokota, Y. Uesu and T. Fukui, Threedimensional observations of polar domain structures using a confocal second-harmonic generation interference microscope, $J$. Appl. Phys. 104, 054112 (2008).

${ }^{22}$ J. Kaneshiro, Y. Uesu and T. Fukui, Visibility of inverted domain structures using the second harmonic generation microscope: Comparison of interference and non-interference cases, J. Opt. Soc. Am. B 27, 888 (2010).

${ }^{23}$ G. D. Boyd and D. A. Kleinman, Parametric interaction of focused Gaussian light beams, J. Appl. Phys. 39, 3597 (1968).

${ }^{24} \mathrm{O}$. Norio and I. Takashi, Etching study of microdomains in $\mathrm{LiNbO}_{3}$ single crystals, J. Appl. Phys. 46, 1063 (1975).

${ }^{25}$ N. A. Pertsev and A. L. Kholkin, Subsurface nanodomains with in-plane polarization in uniaxial ferroelectrics via scanning force microscopy, Phys. Rev. B 88, 174109 (2013).

${ }^{26}$ A. K. Tagantsev, L. E. Cross and J. Fousek, Domains in Ferroic Crystals and Thin Films (Springer, New York, 2010).

${ }^{27}$ O. F. Schirmer, O. Thiemann and M. Woehlecke, Defects in $\mathrm{LiNbO}_{3}$-I. Experimental aspects, J. Phys. Chem. Solids 52, 185 (1991).

${ }^{28}$ V. Gopalan, V. Dierolf and D. A. Scrymgeour, Defect-domain wall interactions in trigonal ferroelectrics, Annu. Rev. Mater. Res. 37, 449 (2007).

${ }^{29}$ L.-H. Peng, Y.-C. Zhang and Y.-C. Lin, Zinc oxide doping effects in polarization switching of lithium niobate, Appl. Phys. Lett. 78, 4 (2001). 